\title{
RESEARCH OF MAGNETIC FIELD DISTRIBUTION IN THE WORKING AREA OF DISK SEPARATOR, TAKING INTO ACCOUNT AN INFLUENCE OF MATERIALS OF PERMANENT MAGNETS
}

\author{
Iryna Shvedchykova \\ Department of Energy management and Applied Electronics \\ Kyiv National University of Technologies and Design (KNUTD) \\ 2 S. Nemyrovycha-Danchenka str., Kyiv, Ukraine, 01011 \\ ishved@i.ua \\ Inna Melkonova ${ }^{1}$ \\ inna.mia.lg@gmail.com \\ Julia Romanchenko ${ }^{1}$ \\ romanchenkojulia321123@gmail.com \\ ${ }^{1}$ Department of Electrical Engineering \\ Volodymyr Dahl East Ukrainian National University \\ 59a S. Central ave., Severodonetsk, Ukraine, 93400
}

\begin{abstract}
Based on the results of a numerical-field analysis of the distribution of the magnetic force field in the working area of the disk magnetic separator, designed to clean bulk substances from ferromagnetic inclusions, the influence of the magnetic material of the poles of the magnetic system on the field distribution is determined. A consistent study of two magnetic systems assembled on the basis of magnetic materials of different classes is carried out. The finite element method implemented in the COMSOL Multiphysics software environment is used to calculate the distribution of magnetic induction in a disk magnetic separator with rare-earth and ferrite magnets. Due to the complexity of the spatial geometry of the force field in the working area of the disk magnetic separator, a three-dimensional model of the magnetic system is developed. A comparative analysis of the distribution of the magnetic force field in the working area of the disk separator with a highly coercive magnetic system and with a magnetic system based on ferrite blocks is carried out. As a result of the analysis, it is found that the indicators of the intensity and heterogeneity of the magnetic field for a highly coercive magnetic system significantly exceed the corresponding parameters of a ferrite magnetic system. It is proved that when choosing magnets for the magnetic system of a disk separator, preference should be given to highly coercive alloys, the magnetic properties of which significantly exceed the magnetic properties of ferrite magnets. To reduce the cost of the magnetic system of the disk separator, the use of a combined magnetic system assembled from magnetic materials of different classes is proposed. Studies of combined magnetic systems with various mass fractions of magnetic materials are done. The ratio of the mass fractions of magnets of various properties in the poles of the magnetic system is determined, at which sufficiently high magnetic characteristics are provided in the working area. It is shown that the presence of a ferrite fraction in the magnetic poles not only reduces the cost of the magnetic system of the separator, but also reduces the mass of the system. The tasks of further research are justified.
\end{abstract}

Keywords: disk separator, magnetic induction, permanent magnet, combined magnets, ferromagnetic inclusions.

DOI: $10.21303 / 2461-4262.2020 .001106$

\section{Introduction}

The widespread use of magnetic separators for cleaning bulk materials from ferromagnetic inclusions, primarily in the food and related industries, actualizes the task of increasing their efficiency, which is understood to ensure maximum reliability of removal. One of the ways to increase the efficiency of devices for magnetic separation is the use of permanent magnets [1-4]. To improve the quality of purification of raw materials and finished products from magnetic impurities, the permanent magnets of the separators must have high magnetic properties and stable magnetic parameters. Moreover, the layout of magnetic systems, including using various types of magnetic materials, to ensure the necessary distribution of the magnetic force field in the working area of the device. 
Permanent magnets, in particular ceramic magnets based on barium or strontium ferrites, have been used in magnetic separators for many years to clean bulk substances from ferromagnetic impurities. Practical experience in operating magnetic systems of separators $[5,6]$ has shown that the widespread use of ferrite magnets is due to their relatively low cost, high stability of magnetic properties and corrosion resistance. Thus, the work [5] summarizes the experience of using ferrite magnetic systems of permanent magnet separators in the food, pharmaceutical, animal feed and other related industries for the purification of bulk substances from ferromagnetic inclusions. It has been shown that with sufficiently long-term use of such systems (about 30 years), the decrease in magnetic induction does not exceed $4 \%$. When developing magnetic systems for pulley separators, as shown in [6], it is advisable to use ferrite permanent magnets due to their relatively low cost.

In recent years, the construction of magnetic systems for separators has found application of highly coercive rare-earth permanent magnets based on a neodymium-iron-boron (Nd-Fe-B) alloy. Permanent magnets of this alloy are characterized by a sufficiently high residual magnetic induction $B_{r}$ (up to $B_{r}=1.44 \mathrm{~T}$ ), temperature stability (up to $150{ }^{\circ} \mathrm{C}$ ), have a small volume per unit of energy.

The disadvantages of magnets include the low operating temperature of some brands, as well as low corrosion resistance, which is eliminated by coating the surface of the magnets with protective layers of copper, zinc, nickel, chromium [7].

Today, Nd-Fe-B-based magnets have no analogues in magnitude of magnetic induction and its stability over time. It is advisable to use magnetic systems of separators with irreplaceable magnets where it is necessary to improve the quality of cleaning with the minimum dimensions of the separator. So, in [2], the prospects of using magnetic systems based on neodymium magnets for the purification of bulk substances from weakly magnetic impurities at food industry enterprises are justified. In [8], a method is presented for separating small metallic non-ferrous particles from two-component metallic non-ferrous mixtures using a new type of permanent magnet separator. It is also promising to use Nd-Fe-B alloy magnets in magnetic systems for high-gradient separation [9, 10], in particular in biomedicine, for targeted delivery and localization of magnetic nanoparticles in a given area of a biological object $[11,12]$.

When constructing magnetic systems of separators based on permanent magnets, a combination of various types of magnetic materials is also used. According to the results of experimental studies in [13], combined layout solutions of magnetic systems of drum-type separators are proposed, which provide for the use of both ferrite and rare-earth permanent magnets. Ferrite magnet blocks are recommended for magnetizing the extreme rows of Nd-Fe-B alloy magnets and for improving the conditions of the east of ore material from the surface of the drum. Moreover, at a distance of $20 \mathrm{~mm}$ from the surface of the drum, the magnetic induction is $0.2 \mathrm{~T}$, and its gradient is $5 \mathrm{~T} / \mathrm{m}$.

In [14], the characteristics of combined magnets are analyzed, which are assembled from different types of magnets and are considered as a new class of permanent magnets. It is shown that the properties of such magnets are determined by both magnetic properties and the volume content of their components, and by the assembly scheme. It is concluded that the theoretical and experimental studies of combined magnets are promising for the purpose of their practical application.

Thus, as the analysis of publications [1-14] shows, a reasonable choice of material of permanent magnets and layout solutions of magnetic systems is an important stage in the design of new types of magnetic separators. At the same time, the question of the effect of the material of the magnets on the distribution of the magnetic field in the working zones of the magnetically separating devices requires attention.

The aim of research is analysis of the distribution of the magnetic force field in the working area of the disk magnetic separator and determination of the effect of the magnetic material of the poles of the magnetic system on this distribution.

To achieve this aim it is necessary:

1) to conduct a comparative analysis of the distribution of the magnetic force field in the working area of the disk separator for various types of magnetic materials of the poles of the magnetic system;

2) to substantiate the feasibility of using combined permanent magnets in the construction of the magnetic system of the disk separator. 


\section{Materials and methods}

To clean bulk materials transported by belt conveyors from unwanted ferromagnetic impurities, a new design of a disk magnetic separator is designed, shown in Fig. 1. On the surface of the stationary ferromagnetic disk 1 there are permanent magnets 2 located in a spiral at the same distance from each other with alternating polarity of the poles, both in the direction of deployment of the spiral and in the radial direction. In the presence of a non-magnetic rotating discharge disk (not shown in Fig. 1), this configuration of the magnetic system ensures its self-cleaning.

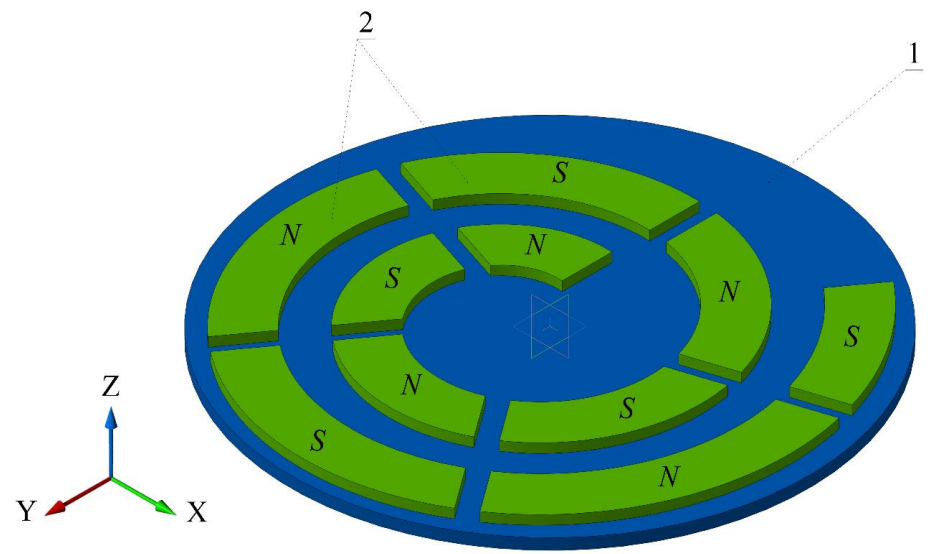

Fig. 1. The magnetic system of the disk separator: 1 - ferromagnetic disk; 2 - permanent magnets

In [15], the results of previous studies of the influence of the spiral geometry of the magnetic system (Fig. 1) on the distribution of the magnetic field in the working region of the separator are presented. An analysis of the spatial distribution of the magnetic field induction in the working area of the magnetic separator, presented in [15], shows that a sufficiently strong magnetic field $\left(B_{\max }=0.6 \ldots 0.65 \mathrm{~T}\right)$ is formed in the working volume of the separator on the surface of the magnets, sufficient for extraction of ferromagnetic inclusions. Studies on the effect of magnetic materials and their layouts on the distribution of the magnetic force field are not conducted.

The magnetic field in a system with permanent magnets in the absence of electric current is described by the Maxwell system of equations, which in the magnetostatic approximation takes the form

$$
\begin{gathered}
\nabla \times \mathbf{H}=0, \\
\nabla \cdot \mathbf{B}=0,
\end{gathered}
$$

where $\mathbf{H}$ - the magnetic field vector; $\mathbf{B}$ - the magnetic induction vector.

Equations for permanent magnets has the form

$$
\mathbf{B}=\mu_{0} \mu_{r} \mathbf{H}+\mathbf{B}_{\mathbf{r}}
$$

where $\mu_{r}, \mathbf{B}_{r}$ - the relative value of the magnetic permeability and residual induction of the permanent magnet material, respectively.

The equation of magnetic state for a ferromagnetic disk (pos. 1, Fig. 1) and the environment (air) can be written as

$$
\mathbf{B}=\mu_{0} \mu_{r} \mathbf{H},
$$

where $\mu_{r}$ - the relative value of the magnetic permeability for the ferromagnetic disk $\left(\mu_{r}=1000\right)$ and air $\left(\mu_{r}=1\right)$, respectively. 
Based on expressions (2)-(4), a differential equation can be obtained for calculating the vector magnetic potential A $(\mathbf{B}=\nabla \times \mathbf{A})$

$$
\nabla\left(\mu_{0} \mu_{r} \nabla A-B_{r}\right)=0
$$

Due to the complexity of the spatial geometry of the force field distribution in the working area of the disk magnetic separator, differential equation (4) is solved using the numerical finite element method in the COMSOL Multiphysics 3.5a 2008 software package.

To solve the first task of conducting a comparative analysis of the distribution of the magnetic force field in the working area of the disk separator using various types of magnetic materials, a computational experiment is conducted. A three-dimensional geometric model of the device's magnetic system with a finite element grid applied to it is shown in Fig. 2, a . When constructing the finite element model, an arbitrary tetrahedral grid is selected. The number of grid points is 18191 , the number of elements is 102,663 , and the number of boundary elements is 10922 , respectively. As the boundary conditions at the external borders of the computational domain, the magnetic isolation condition $A=0$ is used (Fig. $\mathbf{2}, \boldsymbol{b}$ ). At the same time, the continuity condition $A_{1}-A_{2}=0$ is set at the internal boundaries, which is the standard setting of the boundary conditions in the COMSOL Multiphysics software environment.

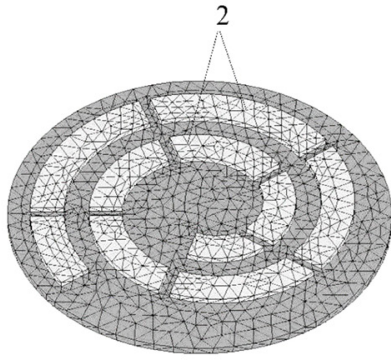

$a$

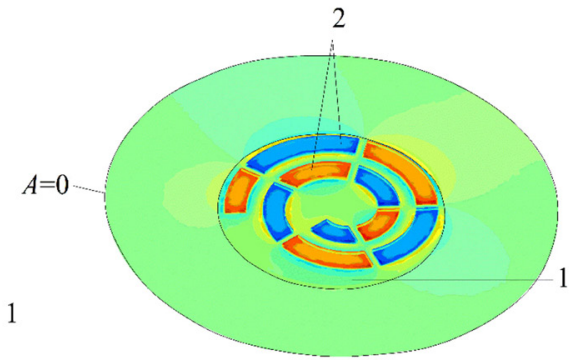

$b$

Fig. 2. Three-dimensional geometric model of the magnetic system

( 1 - ferromagnetic disk, 2 - permanent magnets): $a$ - with a applied grid from finite elements; $b$ - indicating the boundary conditions of the computational domain

In the study, the vertical component of the magnetization of the permanent magnets is directed along the $Z$ axis (Fig. 1). The properties of the permanent magnets are set using equation (2) in the Subdomain Setting module of the COMSOL Multiphysics software environment. This equation makes it possible to better take into account the effects of demagnetization under the action of an external field directed against the vector of the current magnetization.

For ferromagnetic disk 1 (Fig. 1), made of soft magnetic structural steel, the assumption is made that the relative magnetic permeability $\mu_{r}$ of the disk material is constant $\left(\mu_{r}=1000\right)$. The design parameters of the magnetic system of the separator, taken as the base, are (Fig. 3): $\delta=25 \mathrm{~mm}, a=67.6 \mathrm{~mm}, b=51.7 \mathrm{~mm}, t=12.5 \mathrm{~mm}$. The dimensions of the ferromagnetic disk 1 (Fig. 1), on which the permanent magnets are placed, are taken as follows: the disk diameter is $700 \mathrm{~mm}$, and the thickness is $15 \mathrm{~mm}$.

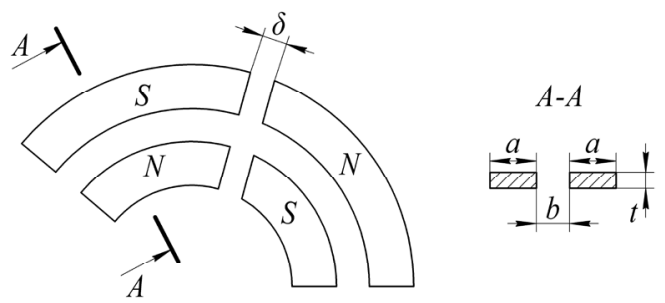

Fig. 3. Design parameters of the magnetic system of the separator 
The influence of the magnetic material of permanent magnets on the distribution of magnetic induction $B$ is studied in an arbitrarily selected air gap of the magnetic system of the separator for characteristic points 1 (or 3) and 2 (Fig. 4) along the vertical $Z$ axis at a distance from the surface of the magnets up to $60 \mathrm{~mm}$. It should be noted that for points 1 and 3, symmetrically located relative to the $Z$ axis, the nature of the distribution of the magnetic field is identical.

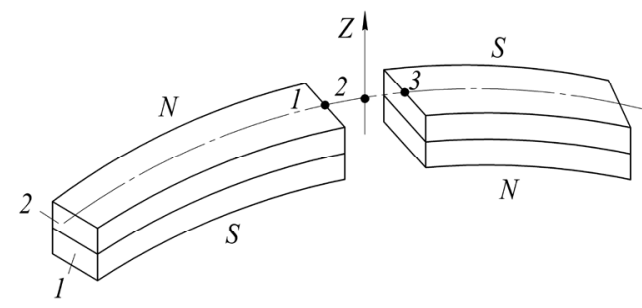

Fig. 4. The location of the characteristic points in the study area:

1 - ferrite magnets; 2 - magnets based on Nd-Fe-B alloy

In the research, it is assumed that the effect of the material is the same for any air gap of the magnetic system located both inside the disk and on its periphery.

\section{Research results of the influence of the material of permanent magnets on the magnetic field distribution}

At the first stage, two magnetic systems assembled on the basis of magnetic materials of different classes are sequentially investigated. For the first magnetic system, magnets based on barium ferrite $\mathrm{Fe}-\mathrm{Ba}$ are chosen (relative magnetic permeability $\mu_{r}=1$, residual magnetic induction $B_{r}=0.4 \mathrm{~T}$, layer 2 in Fig. 3 is absent), for the second - permanent magnets based on highly coercive $\mathrm{Nd}-\mathrm{Fe}-\mathrm{B}$ neodymium-iron-boron alloy (relative magnetic permeability $\mu_{r}=1.06$, residual magnetic induction $B_{r}=1.2 \mathrm{~T}$, layer 1 in Fig. 4 is absent). The results of calculating the vertical component of magnetic induction $B$ (along the vertical axis $Z$ ) for characteristic points 1 and 2 (Fig. 4) are presented in Fig. 5.
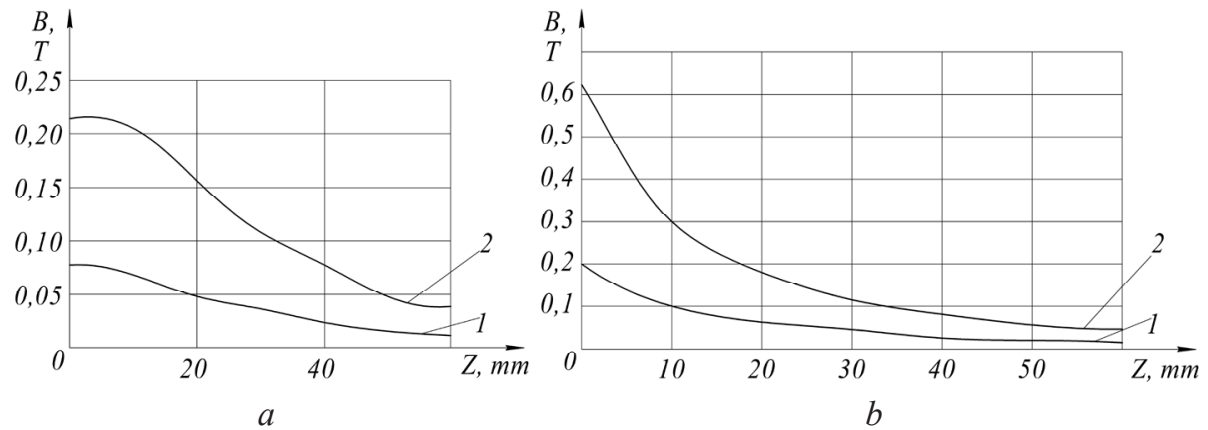

Fig. 5. The distribution of magnetic induction along the vertical $Z$ axis (when constructing a magnetic system based on: 1 - barium ferrite, 2 - based on the Nd-Fe-B alloy): $a$ - for characteristic point $2 ; b$ - for characteristic point 1

To assess the heterogeneity degree of the magnetic field, the modulus of the magnetic induction gradient is $|\operatorname{grad} B|_{i}$ at $i$-th points $(\mathrm{i}=2, \ldots, n, n=6)$. Calculation of $|\operatorname{grad} B|_{i}$ is carried out at three points according to the formula

$$
|\operatorname{grad} B|_{i}=\left|\left(B_{i+1}-B_{i-1}\right) /(2 \nabla Z)\right|
$$

where $\Delta Z$ - the distance between the points where the magnetic induction is determined $(\Delta Z=10 \mathrm{~mm})$.

Magnetic induction gradient modulus calculation results $|\operatorname{grad} B|_{i}$ for characteristic point 2

(Fig. 3) are given in Table 1. 
As can be seen from Fig. 4, the magnetic system based on the Nd-Fe-B alloy creates a magnetic field that is $2.75 \ldots 4$ times higher than the magnetic field intensity from ferrite magnets. Higher induction gradient $|\operatorname{grad} B|_{i}$ values should also be indicated in the working area of the magnetic system. For characteristic point 2, the gradient of magnetic induction $|\operatorname{grad} B|_{i}$ for poles based on Nd-Fe-B alloy exceeds, on average, 2 ... 4.3 times the corresponding indicator for ferrite magnets. These results are in good agreement with the results of studies obtained by other authors [2].

Table 1

$|\operatorname{grad} B|_{i}$ calculation results for characteristic point 2

\begin{tabular}{|c|c|c|c|c|c|}
\hline \multirow{2}{*}{$\mathrm{Z}, \mathbf{m m}$} & \multirow{2}{*}{$\begin{array}{l}\text { Number of the } \\
\text { point } i\end{array}$} & \multicolumn{2}{|c|}{ Nd-Fe-B alloy magnets } & \multicolumn{2}{|c|}{ Barium ferrite magnets } \\
\hline & & $\mathbf{B}, \mathbf{T}$ & $|\operatorname{gradB}|_{\mathrm{i}}, \mathbf{T} / \mathbf{m}$ & $\mathbf{B}, \mathbf{T}$ & $|\operatorname{gradB}|_{\mathrm{i}}, \mathbf{T} / \mathbf{m}$ \\
\hline 0 & 1 & 0.220 & & 0.080 & \\
\hline 10 & 2 & 0.210 & 3 & 0.070 & 1.5 \\
\hline 20 & 3 & 0.160 & 5 & 0.051 & 1.6 \\
\hline 30 & 4 & 0.110 & 4 & 0.038 & 1.25 \\
\hline 40 & 5 & 0.080 & 3 & 0.025 & 1.15 \\
\hline 50 & 6 & 0.050 & 3 & 0.015 & 0.7 \\
\hline 60 & 7 & 0.040 & & 0.011 & \\
\hline
\end{tabular}

Thus, when choosing magnets for the magnetic system of a disk separator, preference should be given to the Nd-Fe-B alloy, the magnetic properties of which significantly exceed the magnetic properties of ferrite magnets.

At the next stage, the magnetic systems of a separator of a combined type, built on the basis of magnets of different classes (combined magnets), are investigated. The layers of magnetic materials at the poles of the magnetic system are arranged as shown in Fig. 3: ferrite magnets are adjacent to the surface of the magnetic disk (Fig. 1) (layer 1 in Fig. 2), and the upper layer of the poles contains a highly coercive Nd-Fe-B alloy (layer 2 in Fig. 2). With this alternation of layers of magnetic materials, a certain magnetization of the upper layer of magnets from the $\mathrm{Nd}-\mathrm{Fe}-\mathrm{B}$ alloy occurs.

Magnetic induction is determined for characteristic points 1 and 2 (Fig. 3) along the vertical $Z$ axis at a distance from the surface of the magnets to $60 \mathrm{~mm}$, starting with a fraction of ferrite magnets of $50 \%$ in each individual pole of the magnetic system. After that, the proportion of ferrite magnets gradually decreases, while the magnitude of the magnetic induction in the working gap of the combined magnet approaches the corresponding parameters of the magnetic system assembled entirely on the basis of the Nd-Fe-B alloy.

The results of calculating the magnetic induction $B$ along the vertical $Z$ axis for combined magnets compared to magnets based on the Nd-Fe-B alloy for point 1 are given in Table 2, and for point 2 - in Table 3, respectively.

As the Tables 2, 3 show, the intensity of the magnetic field in the working zone of the separator depends on the proportion of ferrite magnets in the total mass of the combined magnetic system. So, with a ferrite magnet fraction of $50 \%$, magnetic induction in the corner zone on the surface of a permanent magnet (point 1 or 3 in Fig. 3) is even equal to the magnetic induction that the city has when using highly coercive blocks of magnets, and amounts to $0.62 \mathrm{~T}$. At the same time, at a distance from the surface of the poles magnetic induction is significantly reduced.

With a proportion of ferrite magnets in the total mass of $25 \%$, combined magnets are inferior to Nd-Fe-B magnets at distances up to $40 \mathrm{~mm}$. At the same time, at large distances (50 and $60 \mathrm{~mm}$ ), such arrangements create the same magnesium field as Nd-Fe-B magnets. When the proportion of ferrite magnets in the total mass of the magnetic field is about $18-20 \%$, the magnetic 
induction in the working area of the magnetic system with combined magnets approaches the values of magnetic induction in the Nd-Fe-B magnetic system.

Table 2

The results of calculating the magnetic induction $B$ of the combined magnets compared to magnets based on the Nd-Fe-B alloy for point 1

\begin{tabular}{|c|c|c|c|c|}
\hline \multirow{3}{*}{$\mathbf{Z}, \mathbf{m m}$} & \multicolumn{4}{|c|}{ Magnetic induction $B, T$} \\
\hline & \multirow{2}{*}{ Nd-Fe-B alloy magnets } & \multicolumn{3}{|c|}{ Combined magnets with a share of ferrite magnets in the total mass of the magnetic field, $\%$} \\
\hline & & 50 & 25 & 18 \\
\hline 0 & 0.620 & 0.620 & 0.620 & 0.620 \\
\hline 10 & 0.300 & 0.200 & 0.240 & 0.300 \\
\hline 20 & 0.180 & 0.120 & 0.150 & 0.160 \\
\hline 30 & 0.110 & 0.080 & 0.100 & 0.100 \\
\hline 40 & 0.080 & 0.050 & 0.070 & 0.065 \\
\hline 50 & 0.050 & 0.030 & 0.050 & 0.050 \\
\hline 60 & 0.040 & 0.020 & 0.040 & 0.040 \\
\hline
\end{tabular}

Table 3

The results of calculating the magnetic induction $B$ of the combined magnets compared to magnets based on the Nd-Fe-B alloy for point 2

\begin{tabular}{ccccc}
\hline & & \multicolumn{3}{c}{ Magnetic induction $\mathbf{B}, \mathbf{T}$} \\
\cline { 2 - 4 } $\mathbf{Z}, \mathbf{m m}$ & & Combined magnets with a share of ferrite magnets in the total mass of the magnetic field, $\%$ \\
\cline { 3 - 5 } & & $\mathbf{5 0}$ & $\mathbf{2 5}$ & $\mathbf{1 8}$ \\
\hline 0 & 0.220 & 0.160 & 0.210 & 0.220 \\
10 & 0.210 & 0.150 & 0.200 & 0.210 \\
20 & 0.160 & 0.110 & 0.150 & 0.150 \\
30 & 0.110 & 0.080 & 0.100 & 0.100 \\
40 & 0.080 & 0.050 & 0.070 & 0.070 \\
50 & 0.050 & 0.030 & 0.050 & 0.050
\end{tabular}

Thus, the research results show that when constructing magnetic systems for disk separators, it is advisable to use combined magnets with such a fraction of the ferrite component in the total mass of poles of the magnetic system that does not exceed $20 \%$. Using this arrangement will also result in:

- to reduce the mass of the magnetic system of the separator by $10 \%$ (the calculated mass value for the magnetic system based on Nd-Fe-B is $14.72 \mathrm{~kg}$, and for the combined magnetic system - $13.25 \mathrm{~kg}$, respectively);

- to reduce the cost of the magnetic system of the separator by $25 \%$ (the estimated cost of the magnetic system based on Nd-Fe-B is 880 USD, and the combined magnetic system - 660 USD, respectively).

\section{Discussion of research results}

A comparative analysis of the distribution of the magnetic force field in the working area of the newly designed disk separator makes it possible to quantitatively evaluate the ad- 
vantages of a highly coercive magnetic system over a magnetic system based on ferrite blocks. The main indicators characterizing the efficiency of the magnetic separator, that is, its ability to reliably remove and retain ferromagnetic inclusions, are indicators of the intensity and heterogeneity of the magnetic field in the working area. As calculations show, these indicators for a highly coercive magnetic system significantly exceed the corresponding parameters of a ferrite magnetic system.

In recent years, there has been a downward trend in the cost of rare earth magnets. However, the cost of ferrite magnets still remains significantly less than the cost of magnets based on a neodymium-iron-boron alloy. Therefore, to reduce the cost of the magnetic system of the disk separator, it is proposed to use a combined magnetic system assembled from magnetic materials of different classes. At the same time, it is important to select such a ratio of mass fractions of magnets of various properties in the poles of the magnetic system, at which sufficiently high magnetic characteristics are provided in the working area. In the research process, such a ratio is found. The presence of a ferrite fraction in the magnetic poles not only reduces the cost of a quarter of the magnetic system of the separator, but also reduces the mass of the system.

It should be noted that ferrite magnets of complex shape are not produced due to the difficulties of their manufacture. Therefore, certain difficulties may arise in the manufacture of ferrite poles of sector-like shape adopted in a disk separator. To justify the feasibility of using simpler forms of magnetic poles, for example, prismatic, additional studies of the distribution of the magnetic field in the working area of the magnetic separator are necessary.

\section{Conclusions}

When choosing magnets to build the magnetic system of a disk separator, preference should be given to the Nd-Fe-B alloy, the magnetic properties of which significantly exceed the magnetic properties of ferrite magnets. So, the magnetic system of a disk separator based on the Nd-Fe-B alloy creates a magnetic field that is $2.75 \ldots 4$ times higher than the magnetic field intensity from ferrite magnets. The magnetic induction gradient for a system based on the Nd-Fe-B alloy exceeds, on average, $2 . . .4 .3$ times the corresponding indicator for ferrite magnets.

To reduce the cost of the design of the disk magnetic separator, it is advisable to use combined magnets with a fraction of the ferrite component in the total mass of the poles of the magnetic system not higher than $20 \%$. This arrangement of magnetic materials will lead to a decrease in the mass of the magnetic system of the separator by $10 \%$ and to a decrease in its cost by $25 \%$.

\section{References}

[1] Li, Y., Yang, F. (2016). Research Progress and Development Trend of Permanent Magnetic Separators in China and Abroad. DEStech Transactions on Engineering and Technology Research. doi: https://doi.org/10.12783/dtetr/icvme2016/4873

[2] Slusarek, B., Zakrzewski, K. (2012). Magnetic properties of permanent magnets for magnetic sensors working in wide range of temperature. Przeglad elektrotechniczny, 88 (7), 123-126.

[3] Dimova, T., Aprahamian, B., Marinova, M. (2019). Research of the Magnetic Field Inside a Drum Separator With Permanent Magnets. 2019 16th Conference on Electrical Machines, Drives and Power Systems (ELMA). doi: https://doi.org/10.1109/ elma.2019.8771679

[4] Hisayoshi, K., Uyeda, C., Terada, K. (2016). Magnetic separation of general solid particles realised by a permanent magnet. Scientific Reports, 6 (1). doi: https://doi.org/10.1038/srep38431

[5] Meshcheryakov, I. (2018). Spetsifika vybora magnitnyh materialov dlya magnitnyh separatorov. Kombikorma, 2, $22-24$.

[6] Sayko, O. P., Drobchenko, V. I., Kofanov, A. S., Podolyuh, S. M. (2012). Shkivnye zhelezootdeliteli na postoyannyh magnitah. Ugol' Ukrainy, 7, 43-45.

[7] Romanyshyn, T. L. (2013). Obgruntuvannia vyboru materialu postiynykh mahnitiv dlia lovylnykh prystroiv. Rozvidka ta rozrobka naftovykh i hazovykh rodovyshch, 1 (46), 143-152.

[8] Lungu, M. (2009). Separation of small nonferrous particles using a two successive steps eddy-current separator with permanent magnets. International Journal of Mineral Processing, 93 (2), 172-178. doi: https://doi.org/10.1016/j.minpro.2009.07.012

[9] Zeng, S., Zeng, W., Ren, L., An, D., Li, H. (2015). Development of a high gradient permanent magnetic separator (HGPMS). Minerals Engineering, 71, 21-26. doi: https://doi.org/10.1016/j.mineng.2014.10.009 
[10] Gómez-Pastora, J., Xue, X., Karampelas, I. H., Bringas, E., Furlani, E. P., Ortiz, I. (2017). Analysis of separators for magnetic beads recovery: From large systems to multifunctional microdevices. Separation and Purification Technology, 172, 16-31. doi: https://doi.org/10.1016/j.seppur.2016.07.050

[11] Karlov, A., Kondratenko, I., Kryshchuk, R., Rashchepkin, A. (2014). Magnetic system with permanent magnets for localization magnetic nanoparticles in a given region of the biological environments. Elektromekhanichni i enerhozberihaiuchi systemy, 4, 79-85.

[12] Chen, H., Bockenfeld, D., Rempfer, D., Kaminski, M. D., Liu, X., Rosengart, A. J. (2008). Preliminary 3-D analysis of a high gradient magnetic separator for biomedical applications. Journal of Magnetism and Magnetic Materials, 320 (3-4), $279-284$. doi: https://doi.org/10.1016/j.jmmm.2007.06.001

[13] Myazin, V., Kilin, V., Yakubaylik, E. (2010). Perfection of methods and technology of highly magnetic iron ore concentration in connection with the innovations of the last years (by the example of the Siberian region). Vestnik ChitGU, 6 (63), 95-101.

[14] Kravchenko, A. Y., Bovda, A. M. (2003). Combined permanent magnets: expansion of permanent magnets classification. Elektrotekhnika i Elektromekhanika, 3, 37-39.

[15] Gerlici, J., Shvedchikova, I. A., Nikitchenko, I. V., Romanchenko, J. A. (2017). Investigation of influence of separator magnetic system configuration with permanent magnets on magnetic field distribution in working area. Electrical Electrical Engineering \& Electromechanics, 2, 13-17. doi: https://doi.org/10.20998/2074-272x.2017.2.02 